\title{
A novel framework for MR image segmentation and quantification by using MedGA
}

Leonardo Rund $\coprod^{\dagger *}$ E-mail: leonardo.rundo@disco.unimib.it; lr495@cam.ac.uk

University of Milano-Bicocca, Department of Informatics, Systems and Communication, 20126 Milan, Italy

Institute of Molecular Bioimaging and Physiology, Italian National Research Council, 90015 Cefalù (PA), Italy

University of Cambridge, Department of Radiology, CB2 OQQ Cambridge, United Kingdom

Cancer Research UK Cambridge Centre, CB2 ORE Cambridge, United Kingdom

Andrea Tangherloni ${ }^{\dagger}$, E-mail: andrea.tangherloni@disco.unimib.it

University of Milano-Bicocca, Department of Informatics, Systems and Communication, 20126 Milan, Italy

University of Cambridge, Department of Haematology, CB2 OXY Cambridge, United Kingdom

Wellcome Trust Sanger Institute, Wellcome Trust Genome Campus, CB10 1SA Hinxton, United Kingdom

Paolo Cazzaniga, E-mail: paolo.cazzaniga@unibg.it

University of Bergamo, Department of Human and Social Sciences, 24129 Bergamo, Italy

SYSBIO.IT Centre of Systems Biology, 20126 Milan, Italy

Marco S. Nobile, E-mail: nobile@disco.unimib.it

University of Milano-Bicocca, Department of Informatics, Systems and Communication, 20126 Milan, Italy

SYSBIO.IT Centre of Systems Biology, 20126 Milan, Italy

Giorgio Russo, E-mail: giorgio.russo@ibfm.cnr.it

Institute of Molecular Bioimaging and Physiology, Italian National Research Council, 90015 Cefalù (PA), Italy

Maria Carla Gilardi, E-mail: mariacarla.gilardi@ibfm.cnr.it

Institute of Molecular Bioimaging and Physiology, Italian National Research Council, 90015 Cefalù (PA), Italy

Salvatore Vitabile, E-mail: salvatore.vitabile@unipa.it

University of Palermo, Department of Biomedicine, Neuroscience and Advanced Diagnostics, 90127 Palermo, Italy

Giancarlo Mauri, E-mail: mauri@disco.unimib.it

University of Milano-Bicocca, Department of Informatics, Systems and Communication, 20126 Milan, Italy

SYSBIO.IT Centre of Systems Biology, 20126 Milan, Italy

Daniela Besozzi, E-mail: daniela.besozzi@unimib.it

University of Milano-Bicocca, Department of Informatics, Systems and Communication, 20126 Milan, Italy

Carmelo Militello, E-mail: carmelo.militello@ibfm.cnr.it

Institute of Molecular Bioimaging and Physiology, Italian National Research Council, 90015 Cefalù (PA), Italy

$\dagger$ These authors contributed equally

*Corresponding author. Phone: +44 (0)1223 256255.

Address: Department of Radiology, University of Cambridge, Box 218, Cambridge Biomedical Campus, CB2 0QQ Cambridge, United Kingdom. 


\title{
A novel framework for MR image segmentation and quantification by using MedGA
}

\author{
Leonardo Rundo ${ }^{\dagger, \mathrm{a}, \mathrm{b}, \mathrm{c}, \mathrm{d}, *}$, Andrea Tangherloni ${ }^{\dagger, \mathrm{a}, \mathrm{e}, \mathrm{f}}$, Paolo Cazzaniga ${ }^{\mathrm{g}, \mathrm{h}}$, \\ Marco S. Nobile ${ }^{\mathrm{a}, \mathrm{h}}$, Giorgio Russo ${ }^{\mathrm{b}}$, Maria Carla Gilardi ${ }^{\mathrm{b}}$, Salvatore Vitabile ${ }^{\mathrm{i}}$, \\ Giancarlo Mauri ${ }^{\mathrm{a}, \mathrm{h}}$, Daniela Besozzi ${ }^{\mathrm{a}}$, Carmelo Militello ${ }^{\mathrm{b}}$ \\ ${ }^{a}$ University of Milano-Bicocca, Department of Informatics, Systems and Communication, \\ Milan, Italy \\ ${ }^{b}$ Institute of Molecular Bioimaging and Physiology, Italian National Research Council, \\ Cefalù (PA), Italy \\ ${ }^{c}$ University of Cambridge, Department of Radiology, Cambridge, UK \\ ${ }^{d}$ Cancer Research UK Cambridge Centre, Cambridge, United Kingdom \\ ${ }^{e}$ University of Cambridge, Department of Haematology, Cambridge, UK \\ ${ }^{f}$ Wellcome Trust Sanger Institute, Wellcome Trust Genome Campus, Hinxton, UK \\ ${ }^{g}$ University of Bergamo, Department of Human and Social Sciences, Bergamo, Italy \\ ${ }^{h}$ SYSBIO.IT Centre of Systems Biology, Milan, Italy \\ ${ }^{i}$ University of Palermo, Department of Biomedicine, Neuroscience and Advanced \\ Diagnostics, Palermo, Italy
}

\begin{abstract}
Background and Objectives: Image segmentation represents one of the most challenging issues in medical image analysis to distinguish among different adjacent tissues in a body part. In this context, appropriate image pre-processing tools can improve the result accuracy achieved by computer-assisted segmentation methods. Taking into consideration images with a bimodal intensity distribution, image binarization can be used to classify the input pictorial data into two classes, given a threshold intensity value. Unfortunately, adaptive thresholding techniques for two-class segmentation work properly only for images characterized by bimodal histograms. We aim at overcoming these limitations and automatically determining a suitable optimal threshold for bimodal Magnetic Resonance (MR) images, by designing an intelligent image analysis framework
\end{abstract}

* Corresponding author.

E-mail address: leonardo.rundo@disco.unimib.it; lr495@cam.ac.uk (L. Rundo)

${ }^{\dagger}$ These authors contributed equally. 
tailored to effectively assist the physicians during their decision-making tasks.

Methods: In this work, we present a novel evolutionary framework for image enhancement, automatic global thresholding and segmentation, which is here applied to different clinical scenarios involving bimodal MR image analysis: $(i)$ uterine fibroid segmentation in MR guided Focused Ultrasound Surgery, and (ii) brain metastatic cancer segmentation in neuro-radiosurgery therapy. Our framework exploits MedGA as a pre-processing stage. MedGA is an image enhancement method based on Genetic Algorithms that improves the threshold selection, obtained by the efficient Iterative Optimal Threshold Selection algorithm, between the underlying sub-distributions in a nearly bimodal histogram.

Results: The results achieved by the proposed evolutionary framework were quantitatively evaluated, showing that the use of MedGA as a pre-processing stage outperforms the conventional image enhancement methods (i.e., histogram equalization, bi-histogram equalization, Gamma transformation, and sigmoid transformation), in terms of both MR image enhancement and segmentation evaluation metrics.

Conclusions: Thanks to this framework, MR image segmentation accuracy is considerably increased, allowing for measurement repeatability in clinical workflows. The proposed computational solution could be well-suited for other clinical contexts requiring MR image analysis and segmentation, aiming at providing useful insights for differential diagnosis and prognosis.

Keywords: Image pre-processing, Adaptive thresholding, Quantitative medical imaging, Evolutionary Computation, Magnetic Resonance Imaging, Bimodal intensity distribution

\section{Introduction}

Medical image segmentation concerns both detection and delineation of anatomical or physiological structures from the background, distinguishing among the different components included in the image [1]. This important task allows

5 for the extraction of clinically useful information and features in medical im- 
age analysis [2, 3]. Accordingly, computer-assisted approaches enable quantitative imaging [4], whose aim is to derive accurate and objective measurements from digital images regarding a Region of Interest (ROI) [5, 6. Indeed, image segmentation is still one of the most compelling research areas, especially in medical image analysis [4, in which accurately delineating the ROIs is a critical task, since manual segmentation procedures are time-expensive, error-prone, and operator-dependent (i.e., they do not guarantee result repeatability).

In Pattern Recognition, among the low-level intensity-based techniqueswhich are widely adopted in scenarios with real-time constraints - the most basic unsupervised image segmentation approach is global thresholding that essentially reduces to a pixel classification problem [7. In particular, image binarization classifies the input pictorial data into exactly two classes (i.e., foreground and background), given a threshold intensity value [8. This global threshold value is efficiently computed by operating on the image histogram alone. Unfortunately, image binarization techniques work properly only for input images characterized by a bimodal histogram [9, while in practice different types of regions in an image could overlap, thus affecting the bimodality conditions of the gray level histogram, where the histogram modes semantically correspond to different types of regions. Image pre-processing can definitely 25 improve the accuracy of computer-assisted segmentation methods [8], by sharpening the peaks of the two sub-distributions, so that the resulting histogram is characterized by a stronger bimodality, even in the case of blurred region contours and of the related Mach band effect pertaining to edge-detection in the human visual system [10, 11. As a way of example, in radiology this phenomenon is accentuated in the edges of adjacent regions that slightly differ in terms of gray level intensities $[12$.

No existing pre-processing technique addresses the issues related to medical image enhancement for subsequent binarization using adaptive thresholding [9]. Literature methods may be inadequate when dealing with low-contrast images 35 [13, producing false edges and under-/over-segmentation when input images are affected by noise, as in the case of Magnetic Resonance Imaging (MRI) [14]. 
This modality represents the most common soft-tissue acquisition technique in current medical practice, with particular relevance in oncological imaging, allowing for high-contrast between the tumors and the surrounding tissues [15].

40 Unfortunately, MRI data are affected by acquisition noise [16] and are also prone to imaging artifacts, mainly caused by magnetic susceptibility and large intensity inhomogeneities of the principal field (i.e., streaking or shadowing artifacts [17]), especially in the latest MRI acquisition devices with high magnetic field intensity. In order to overcome these limitations and automatically determine a 45 suitable optimal threshold, in this work, we present a novel framework for image enhancement, automatic global thresholding and segmentation, where the pre-processing stage is realized by means of MedGA [18], an intelligent image enhancement method based on Genetic Algorithms (GAs) that improves the threshold selection, obtained by the efficient Iterative Optimal Threshold Selection (IOTS) algorithm [19, 20, between the underlying sub-distributions in a nearly bimodal histogram, so effectively assisting the physicians during their decision-making tasks. GAs have been previously employed in this field for different purposes, as in the case of [21], where the optimization capabilities of GAs were exploited to identify the best parameters of a de-noising filter applied to brain MR images.

In this paper, we propose a complete framework (graphically represented in Fig. 11 that is not limited to the enhancement of MR images, but covers all the steps required for image analysis, including the radiology reporting phase. In more detail, every processing step is taken into account, from cropping (given a bounding region containing an image portion characterized by a bimodal intensity distribution), normalization (also by means of interactive windowing) and enhancement of MR images, to the application of an efficient thresholdingbased segmentation algorithm for quantification purposes. Indeed, our final goal is the definition of a computational tool well-suited for several clinical contexts, capable of providing useful insights for differential diagnosis and prognosis.

In particular, this computational approach is here applied to two different clinical scenarios involving contrast-enhanced (CE) MR image analysis: 


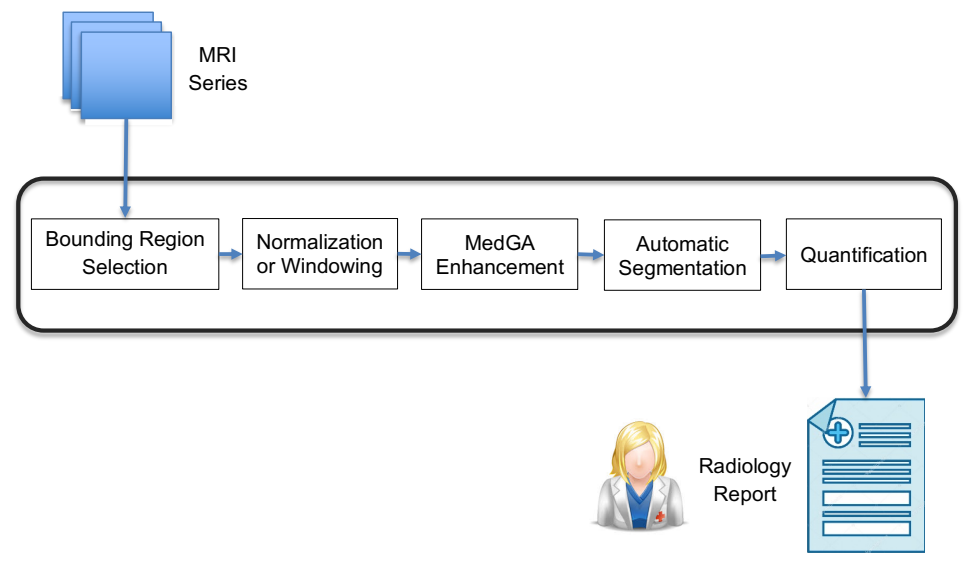

Figure 1: Integration of the proposed computational framework within the context of a clinical scenario, aiming at pointing out its relevance in practical medical applications. All the steps required for medical image analysis, including the quantification and radiology reporting phase, are considered.

- uterine fibroid segmentation in Magnetic Resonance guided Focused Ultrasound Surgery (MRgFUS) [22];

- brain metastatic cancer segmentation in neuro-radiosurgery therapy [23.

In both cases, a precise, reliable and reproducible segmentation is mandatory, whereas these tasks are generally carried out manually by experienced physicians. We highlight the advantages of our evolutionary framework by quantitatively comparing the results achieved by exploiting MedGA against the most common image pre-processing methods (i.e., histogram equalization, bihistogram equalization, Gamma transformation, and sigmoid transformation), in terms of both MR image enhancement and segmentation.

The manuscript is structured as follows. The state-of-the-art of image preprocessing methods is presented in Section 2, while the theoretical aspects of so GAs and image thresholding are explained in Section 3 Section 4 introduces the analyzed MRI datasets, as well as the proposed pre-processing application of MedGA. Section 5 shows the achieved experimental results on the analyzed MR images. Some discussions and conclusions are provided in Section 6 . 


\section{Background}

of abstraction - wherein both input and output are gray-scale images - based on intensity transformations. These global transformations just deal with the gray levels, without considering the pixel position, and the corresponding relationship with its neighborhood in the image. The main objective of pre-processing is to some image features important for further processing [24, 25].

Conventional pre-processing techniques generally expand a narrow input range of gray levels into a wider range of output levels, to improve the image detection performance. Pre-processing tackles the problems related to image can be made, as well as when neither a priori knowledge about the acquisition device nor the objects of interest are available [24]. In this context, Soft Computing methods could be considerably beneficial by considering, for instance, meta-heuristics [26] or Fuzzy Logic [27] to find the best solution in a high variety of scenarios. More specifically, global search meta-heuristics are required to automatically identify the best solution case-by-case. Considering the Computational Intelligence techniques, GAs are the most appropriate framework because of the discrete encoding of the candidate solutions and the combinatorial structure of the search space. More specifically, here we exploited GAs to efficiently encode the individuals by means of their corresponding image histograms.

In medical imaging, pre-processing steps are valuable for further computerassisted image analysis, by making anatomical or functional structures more easily detectable. Indeed, biomedical images usually require methods tailored on a specific task [28], as in the case of well-designed image pre-processing techniques that can improve the ROI feature detection, which is then exploited in downstream image analysis pipelines. For instance, these pre-processed data are suitable for image segmentation algorithms, as they improve their accuracy. Therefore, pre-processing involving image enhancement operations may 
be needed to achieve more identifiable and sharpened boundaries for medical image segmentation [29]: a better distinction among adjacent tissues in medical images can be achieved, by means of an adequate contrast performing grayscale transformations [30. Naïve smoothing techniques, based on both linear (e.g., average or Gaussian filters) or non-linear (median filter) operators, may improve the shape of the histogram [8], also by widening the separation between the two modes in bimodal gray-level histograms 31. These approaches are generally exploited for noise reduction, even though sharp boundaries might be replaced with a fuzzy region of varying shades of gray. Thus, such a kind of low-pass filtering could affect the actual pictorial content, compromising the clinical effectiveness of the subsequent ROI detection and delineation phases.

Among the most used image enhancement techniques, Histogram Equalization (HE) [7] could be not suitable for medical images due to the obtained over-brightness [14]. This method uniformly spreads the input gray level values according to the cumulative density function of the image histogram. However, HE does not preserve the input mean brightness, possibly suffering from overenhancement and giving rise to artifacts such as washed-out effect 32. This global transformation applies contrast stretching just on gray levels with the highest frequencies, causing a significant contrast loss for gray levels characterized by lower occurrences in the input histogram [33]. In order to address the issues related to input mean brightness preservation, a modification of the standard HE technique, called Bi-Histogram Equalization (Bi-HE), was introduced [33. Bi-HE attempts to improve the results achieved by HE, by first splitting the original histogram into two components according to the global mean of the original image, and then separately performing the HE method on the two sub-histograms.

Other traditional global gray level transformations generally used for contrast stretching are formalized as transformation functions of the form $s=\mathcal{T}(r)$, where $\mathcal{T}(\cdot)$ maps an input intensity value $r$ into an output intensity value $s$. 7 . Power-law transformation - also called Gamma Transformation (GT) - is a nonlinear operation of the form $\mathcal{T}(r)=c r^{\gamma}$, where typically $c=1$. For instance, 
when the image is predominantly dark, an expansion of the intensity levels is desirable. In such a case, GT with $\gamma<1$ yields a brighter image by increasing the number of hyper-intense pixels. On the contrary, by using $\gamma>1$, the GT converts the input gray-scale range into a darker one, by increasing the occurrences of darker pixels. Obviously, the value of $\gamma$ strongly depends on the medical application. Accordingly, logarithmic and anti-logarithmic transformations make an image much brighter and darker, respectively. Unfortunately, for medical images characterized by low-contrast and weak edges at adjacent tissue boundaries, GT may result in merely brighter or darker images, leading to difficulties in the visualization and interpretation of different tissues. Therefore, to adequately enhance contrast, the two different behaviors - corresponding to values $\gamma>1$ and $\gamma<1$ - should be combined for contextually decreasing the darker pixel gray values and increasing the brighter pixel gray values. This results in a significant improvement of the contrast, by enhancing the edges thanks to the increased gradient magnitude of the image [14].

This kind of contrast stretching can be achieved by using a Sigmoid intensity Transformation (ST), which darkens a wide range of hypo-intense gray levels and brightens a wide range of hyper-intense gray levels [7. Such an operation indirectly increases the difference between low and high intensity values, resulting in the overall contrast enhancement of the image [14].

In addition to $\mathrm{HE}$, which automatically yields an image with a uniform histogram, it is possible also to explicitly specify the desired shape of the output histogram. This method, named Histogram Specification (HS), aims at matching the histogram of the gray level intensities of the input image against a desired histogram [7. Unfortunately, such approaches cannot be applied in the case of image datasets with heterogeneous gray level distributions, since the histogram to be matched should be defined either a priori for all the images in the dataset or interactively for each processed image, by separating and shaping the two underlying sub-distributions 34.

Unlike existing image enhancement techniques, here we aimed at the definition of a framework employing an appropriate pre-processing method that is able 
to reliably enhance a particular type of medical images for further automated image processing phase. In particular, MedGA [18 tackles the problems related to medical images with a roughly bimodal histogram, by strengthening the two underlying sub-distributions. The main goal of MedGA is to yield pre-processed medical images well-suited for classic threshold-based segmentation techniques to improve ROI delineation. By so doing, the computational load, required for achieving accurate segmentation results, is transferred from the pixel classification stage to the pre-processing phase by means of an effective Soft Computing technique. Differently, supervised - such as Artificial Neural Networks (ANNs) [35] and Support Vector Machines (SVMs) [36] - as well as unsupervised learning approaches - such as Self-Organizing Maps (SOMs) 37] - require a training phase and are generally exploited in image classification and recognition tasks.

The MR images enhanced with MedGA are finally segmented exploiting an efficient version of the IOTS algorithm [19, 20]. For the sake of completeness, we refer the interested readers to some extensive reviews about segmentation algorithms used in the field of medical images based on biophysical models [38, 39, 40], such as in the case of ear imaging [41], female pelvic cavity [42], or pigmented skin regions [43].

\section{Theory}

This section provides the prerequisite notions related to GAs and image thresholding, in order to understand the rationale and the key concepts concerning this study.

\subsection{Genetic algorithms}

GAs represent an Evolutionary Computation (EC) technique for global optimization tasks. Inspired by Darwin's theory of biological evolution, GAs search optimal solutions to complex problems by evolving a population $P$ of randomly created candidate solutions [44]. In the most general formulation, each "individual" encoded as a fixed length string of characters taken from a finite alphabet (i.e., the genes) represents a solution. 
Relying on a quality measure (i.e., the fitness value), promising individuals are selected according to a specific strategy for the next generation. These individuals undergo crossover and mutation operations to the aim of exchanging and introducing new genetic material in the population, respectively. To prevent the quality of the best solution from decreasing during the iterations, the elitism

210

Several literature methods have been proposed to implement adaptive thresholding methods, able to automatically select a proper value for each analyzed image. The most widespread algorithms for dynamic thresholding are: the 

Otsu 45]; the Minimum-Error Thresholding (MET) method conceived by Kittler and Illingworth [46, later extended by Ye and Danielsson [47. All these approaches are closely related and strongly rely on images characterized by bimodal histograms [9]. In addition, the two populations (i.e., foreground and background pixels in the case of two-class image segmentation), assumed to be nearly Gaussian distributions, should be characterized by approximately equal size and variance [48. When these bimodality assumptions are not satisfied the aforementioned algorithms show some limitations. As a matter of fact, the optimal threshold $\theta_{\text {opt }}$ - especially in the case of Otsu's method-either overor under-estimates the ROI, since the computed threshold tends to split the class with larger size and to bias towards the class with larger variance. Under these conditions, the IOTS method [19, 20] could provide better results than Otsu's method [45] when the sizes of the two classes are highly different 49. Medina-Carnicer et al. [50] also showed that the above mentioned algorithms the case of images affected by intensity overlap, the IOTS algorithm is less likely to either over- or under-estimate the threshold, when compared to other techniques selecting a threshold between the two peaks of the histogram, even if the histogram is not strongly bimodal [24], in particular when applied to medical 255 images [51.

\section{Materials and methods}

In this section, we first present the MRI datasets considered in this work, then we describe the computational framework integrating MedGA for medical image analysis.

\subsection{Clinical MRI datasets}

The proposed pipelines take into account clinical MRI datasets from patients affected by: $(i)$ uterine fibroids; $(i i)$ brain metastatic cancers. All the analyzed 
MRI data are encoded in the 16-bit Digital Imaging and Communications in Medicine (DICOM) format. The MRI acquisition characteristics are reported in Table 1 .

\subsubsection{Uterine fibroids}

Eighteen patients affected by symptomatic uterine fibroids who underwent MRgFUS therapy [52] were considered. The total number of the examined fibroids was 29, overall represented on $163 \mathrm{MR}$ slices, since some patients presented a pathological scenario with multiple fibroids. The analyzed images were acquired using a Signa HDxt 1.5 T MRI scanner (General Electric Medical Systems, Milwaukee, WI, USA) at two different institutions. These MRI series were acquired after the MRgFUS treatment, executed with the ExAblate 2100 (Insightec Ltd., Carmel, Israel) HIFU equipment. The considered MR slices 275 were scanned using the T1-weighted (T1w) "Fast Spoiled Gradient Echo + Fat Suppression + Contrast mean" (FSPGR+FS+C) sequence. This MRI protocol is usually employed for Non-Perfused Volume (NPV) assessment, since ablated fibroids appear as hypo-intense areas due to low perfusion of the contrast mean 22]. Sagittal MRI sections were processed, in compliance with the current clinical routine for therapy response assessment [22. In current clinical practice, the NPV evaluation procedure is fully manual [53. Two uterine fibroid MR slices are depicted in Figs. 2a and 2b.

\subsubsection{Brain metastatic tumors}

Twenty-seven brain metastases treated using a Leksell Gamma Knife (Elekta, Stockholm, Sweden) stereotactic neuro-radiosurgical device [54] were processed, for a total of $248 \mathrm{MR}$ slices. All the available MRI datasets were acquired on a Gyroscan Intera 1.5 T MR Scanner (Philips Medical System, Eindhoven, The Netherlands), before treatment, for the planning phase. In current radiation therapy practice, Gamma Knife treatments are planned manually by a neurosurgeon on MRI alone, by typically using T1w Fast Field Echo (T1w FFE) CE-MRI sequences [55, 56]. Thanks to the Gadolinium-based contrast agent, 
Table 1: MRI acquisition parameters of uterine fibroids and brain metastatic tumors dataset.

\begin{tabular}{c|ccccccc}
\hline \hline Dataset & MRI sequence & TR [ms] & TE [ms] & Matrix size [pixels] & Slice spacing [mm] & Slice thickness [mm] & Pixel spacing [mm] \\
\hline Uterine fibroids & T1w FSPGR+FS+C & $150-260$ & $1.392-1.544$ & $512 \times 512$ & 5.0 & 6.0 \\
Brain metastases & T1w FFE & 25 & $1.808-3.688$ & $256 \times 256$ & 1.5 & $1.6641-0.7031$ \\
\hline \hline
\end{tabular}

brain lesions appear as enhanced hyper-intense zones. Sometimes a dark area might be present due to either edema or necrotic tissues [55, 57]. Two representative instances of brain tumors are shown in Figs. 2c and 2d.

\subsection{The proposed evolutionary framework for bimodal MR image analysis}

The proposed novel evolutionary framework, whose functioning is schematized in Fig. 3, is designed ad hoc for bimodal MR image analysis in order to improve the segmentation and quantification results achieved by basic thresholding techniques. Each processing step is explained in what follows.

\subsubsection{Medical image pre-processing based on GAs}

MedGA requires an input image $\mathcal{I}_{\text {orig }}$, with $M$ rows and $N$ columns, depicting a ROI included in a bounding region whose pixel values are approximately characterized by an underlying bimodal histogram. Therefore, as a first step, either a computational method or a user must detect a region including the ROI (upper part of Fig. 3). Then, the input MR image is masked with this bounding region and the whole image is cropped according to the smallest rectangle enclosing the bounding region. A linear normalization is applied on the initial full range of the masked MR image to balance out the pixel distribution for the following bin rearrangement by means of MedGA. It is worth noting that no additional pre-processing operation (e.g., low-pass or high-pass filtering) is needed by MedGA. The final best solution found by MedGA will emphasize the two underlying bimodal Gaussian distributions occurring in the MR image, for the subsequent image thresholding phase, according to the optimal adaptive threshold $\theta_{\text {opt }}$ computed by means of the efficient IOTS algorithm [19, 20].

The candidate solutions are encoded by MedGA as follows. Let $l_{i n}^{(\min )}$, $l_{\text {in }}^{(\max )}, l_{\text {out }}^{(\min )}$ and $l_{\text {out }}^{(\max )}$ be the minimum non-zero and maximum gray levels 


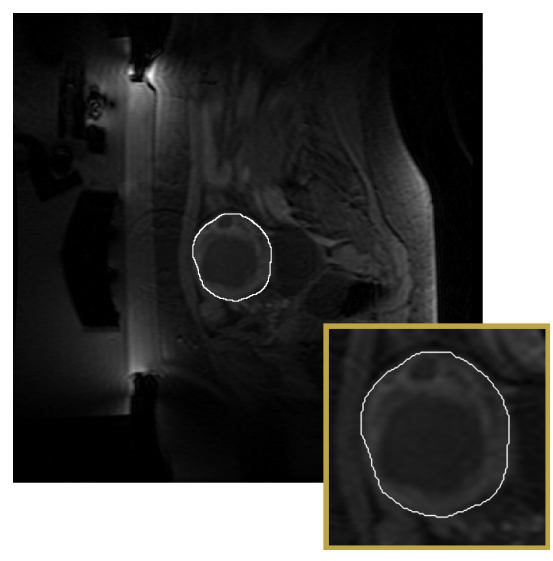

(a)

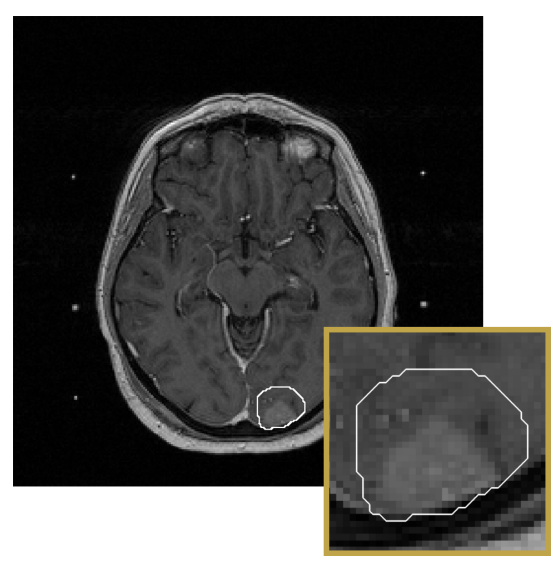

(c)

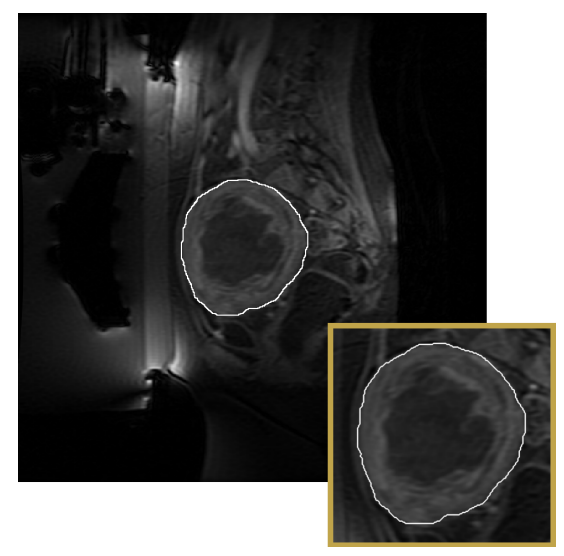

(b)

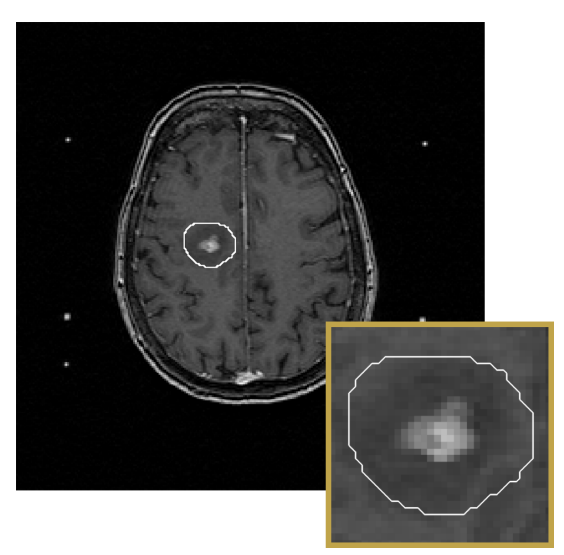

(d)

Figure 2: Examples of input MR images: (a, b) uterine fibroid inside the uterus region; (c, d) brain tumor inside a ROI bounding region selected by the healthcare operator. The image regions including the ROIs, defined by the white contour and zoomed at the bottom right of each sub-figure, are characterized by nearly bimodal histograms.

of input and output images, respectively. Assuming that $l_{\text {in }}^{(\min )} \leq l_{\text {out }}^{(\min )}$ and $l_{\text {in }}^{(\max )} \geq l_{\text {out }}^{(\max )}$, the linear normalization applied to $\mathcal{L}_{\text {in }}=\left[l_{\text {in }}^{(\min )}, \ldots, l_{\text {in }}^{(\max )}\right]$ gives rise to the extended range of the non-zero gray levels, that is, the ordered ${ }_{320}$ set $\mathcal{L}_{i n}^{\prime}=\left[1, \ldots, l_{i n}^{(\max )}\right] \subset \mathbb{N}$ (typically, $l_{i n}^{(\min )}>1$ ). This normalization operation, which employs only values of gray levels already representable in the 


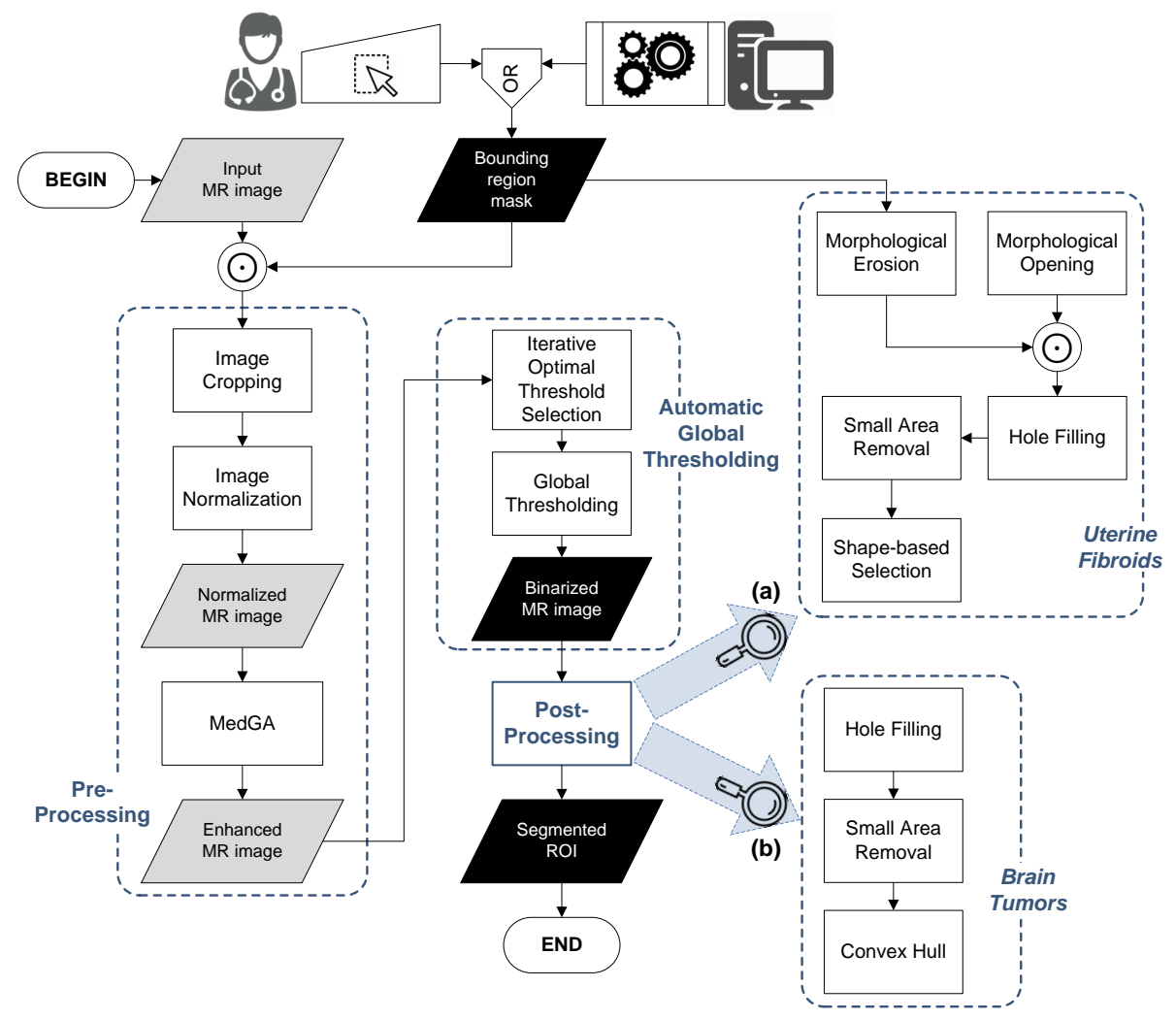

Figure 3: Flow diagram of the proposed computational framework that integrates MedGA [18] as a pre-processing step for MR image segmentation based on the efficient IOTS algorithm 19, 20. Two different post-processing pipelines were developed to deal with MR images of (a) uterine fibroids and (b) brain tumors. Gray and black data blocks denote MR gray-scale images and binary masks, respectively.

initial dynamic range, does not alter the image content and allows MedGA to process additional intensity levels with respect to the initial full range $\mathcal{L}_{i n}$, by considering the variability within the analyzed MRI datasets. During each iteration $t$, with $t=1,2, \ldots, T$, each individual $C_{i}^{t}=\left[C_{i}^{t}(1), C_{i}^{t}(2), \ldots, C_{i}^{t}(n)\right]$ (with $i=1,2, \ldots,|P|$ ) is defined as a circular array of integer numbers of size $n$, where $n=\left|\mathcal{L}_{i n}^{\prime}\right|$ corresponds to the number of different gray levels belonging to $\mathcal{L}_{\text {in }}^{\prime}$ identified in the input MR image (i.e., the gray levels whose frequency is greater than zero in the input MR image). Each individual $C_{i}^{0} \in P$ is randomly 
$\mathcal{L}_{i n}^{\prime}$. The $n$ values are then sorted in ascending order so that the intensity levels $C_{i}^{t}(j)$ (with $j=1,2, \ldots, n$ ), codified by the $i$-th individual, can be mapped to the intensity levels of the input MR image. Stated otherwise, the gray level frequencies of the input MR image are assigned to the corresponding intensity levels of the individual.

In order to evaluate the fitness value of the individuals, we apply the following transformation $\mathcal{T}$ that re-maps each input gray level $r$ into $s$ :

$$
s=\mathcal{T}\left(C_{i}^{t}(j)\right)=\mathcal{T}(r)
$$

where $r \in \mathcal{L}_{\text {in }}=\left[l_{\text {in }}^{(\min )}, \ldots, l_{\text {in }}^{(\max )}\right] \subset \mathbb{N}$ and $s \in \mathcal{L}_{\text {out }}=\left[l_{\text {out }}^{(\min )}, \ldots, l_{\text {out }}^{(\max )}\right] \subset \mathbb{N}$ are intensity values in the input and output gray-scale ranges, respectively. Specifically, $\mathcal{T}$ defines a direct mapping between the gray levels of the original image $\mathcal{I}_{\text {orig }}$ and the output $\mathcal{I}_{\text {enh }}$, namely, each gray level in the original histogram is replaced with the gray level value corresponding to the same position in the final best solution $C_{\text {best }} \in P$.

MedGA uses a tournament selection and a single-point crossover. The mutation strategy [58] is defined as follows: for each gene $j$ (with $j=1,2, \ldots, n$ ) of each offspring, a real number $\rho$ is randomly generated from a uniform distribution in $[0,1]$. If $\rho$ is lower than the mutation rate $p_{m}$, the gene $j$ is mutated as follows:

- if the value of the gene $j$ is less than the optimal threshold $\theta_{\text {opt }}$, then a random integer $\eta_{j}$ is generated in the ordered range $\left[l_{\text {out }}^{(\min )}, \ldots, \theta_{\text {opt }}-1\right]$ and the value of the gene $j$ is replaced with $\eta_{j}$;

- if the value of the gene $j$ is greater than or equal to the optimal threshold $\theta_{\mathrm{opt}}$, then the random integer $\eta_{j}$ is generated in the range $\left[\theta_{\mathrm{opt}}, \ldots, l_{\text {out }}^{(\max )}\right]$ and the value of the gene $j$ is replaced with $\eta_{j}$.

Finally, MedGA also employs an elitism strategy to preserve the best individual of the current population. 
In order to obtain a bimodal histogram separation that allows better results for further automated image processing phases, MedGA exploits a fitness function that aims at realizing two well-separated normal distributions with equal distance from the optimal threshold $\theta_{\text {opt }}$. For each generation $t$ and for each individual $C_{i}^{t}$, first the mean values $\mu_{1, i}^{t}$ and $\mu_{2, i}^{t}$-regarding the two subdistributions in the histogram $\mathcal{H}_{1, i}^{t}$ and $\mathcal{H}_{2, i}^{t}$, respectively - as well as the corresponding optimal threshold $\theta_{\mathrm{opt}, i}^{t}$ are efficiently computed by using the IOTS algorithm [19, 20]. Then, for each individual $C_{i}^{t}$ the following fitness function is calculated:

$$
\begin{aligned}
\mathcal{F}\left(C_{i}^{t}\right) & =\tau_{1}+\tau_{2}+\tau_{3}, \quad \text { where: } \\
\tau_{1} & =\left|2 \cdot \theta_{\mathrm{opt}, \mathrm{i}}^{t}-\mu_{1, i}^{t}-\mu_{2, i}^{t}\right| \\
\tau_{2} & =\left|\omega_{1, i}^{t}-3 \sigma_{1, i}^{t}\right| \\
\tau_{3} & =\left|\omega_{2, i}^{t}-3 \sigma_{2, i}^{t}\right|
\end{aligned}
$$

The terms $\omega_{1, i}^{t}=\frac{1}{2}\left(\theta_{\mathrm{opt}, i}^{t}-\min _{j \in\{1,2, \ldots, n\}}\left\{C_{i}^{t}(j)\right\}\right)$ and $\omega_{2, i}^{t}=\frac{1}{2}\left(\max _{j \in\{1,2, \ldots, n\}}\left\{C_{i}^{t}(j)\right\}-\right.$ $\left.\theta_{\mathrm{opt}, i}^{t}\right)$ correspond to the half width of $\mathcal{H}_{1, i}^{t}$ and $\mathcal{H}_{2, i}^{t}$, respectively, while $\sigma_{1, i}^{t}$ and $\sigma_{2, i}^{t}$ are the standard deviations of $\mathcal{H}_{1, i}^{t}$ and $\mathcal{H}_{2, i}^{t}$, respectively. The three terms of the fitness function $\mathcal{F}(\cdot)$ cooperate together to achieve the desired image enhancement: $\tau_{1}$ aims at maintaining the mean values $\mu_{1, i}^{t}$ and $\mu_{2, i}^{t}$ at the same distance from the yielded optimal threshold $\theta_{\mathrm{opt}, i}^{t}$, while $\tau_{2}$ and $\tau_{3}$ are designed to force the sub-histograms $\mathcal{H}_{1, i}^{t}$ and $\mathcal{H}_{2, i}^{t}$, respectively, to approximate normal distributions $\$ MedGA's performances depend on a proper choice of the functioning settings of the underlying GA; an extensive analysis of the impact of the these values on the outcome of MedGA is presented in [18].

Two examples of image enhancement results, achieved by MedGA on a uterine fibroid and on a brain tumor, are shown in Figs. 4 and 5 , respectively. In the case of uterine fibroids, MedGA enhances the input MR image by making

\footnotetext{
${ }^{1}$ We exploited the empirical 3- $\sigma$ rule, which states that approximately $99.73 \%$ of the values lie within $3 \sigma$ of the mean according to: $\operatorname{Pr}(\mu-3 \sigma \leq X \leq \mu+3 \sigma) \approx 0.9973$, where $\mu, \sigma$ and $X$ represent the mean, the standard deviation and an observation from a normally distributed random variable, respectively.
} 
fibroid regions more uniform and with sharper edges in terms of both visual human perception and automated image segmentation. The histogram in Fig. 4d points out that the output image is characterized by a more defined bimodal distribution compared to the initial image (Fig. 4b), which presents approximately a trimodal gray level distribution. In the case of brain tumors, MedGA enhances the underlying bimodal distribution related to contrast-enhancing tumoral tissue and brain healthy tissues on CE-MR images. This visual achievement is endorsed by the histogram of the enhanced image (Fig. 5d) that shows two more distinct peaks with respect to the initial gray level distribution (Fig. 5b).

\subsubsection{MR image segmentation using adaptive thresholding}

Image enhancement techniques can facilitate the user interpretation of an image as well as improve the automated image understanding. Therefore, we use MR image segmentation as an important processing goal [59. The images enhanced with MedGA are segmented using the IOTS algorithm [19, 20, which is the simplest automated segmentation approach. Our computational framework includes two different post-processing pipelines to refine the results achieved by this efficient adaptive thresholding technique (see Fig. 3). These post-processing steps, here applied to perform uterine fibroid and brain tumor segmentation, are described in what follows. MedGA is able to enhance images in segmentation tasks involving both hyper- and hypo-intense ROIs in CE-MR images, also dealing with data unbalanceness (i.e., the number of foreground pixels is either much higher or lower than the number of background pixels). The thresholding-based segmentation results achieved on the MR images preprocessed by MedGA (Figs. 4c and 5c are shown in Figs. 6a and 6b.

Uterine fibroid segmentation. First, uterus region delineation is required; this task can be accomplished manually by the user or automatically by means of computational methods to reduce operator-dependency, as described in [60]. ROIs are represented by tissues with low contrast mean absorption (i.e., NPV), thus the pixels with lower values with respect to the achieved threshold are yielded in the binarized MR image. However, segmentation approaches have 


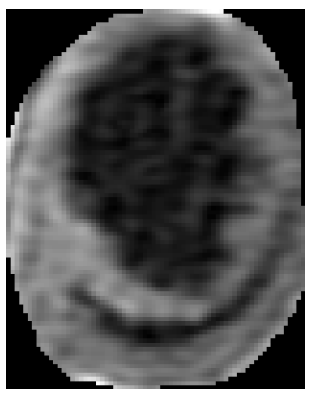

(a)

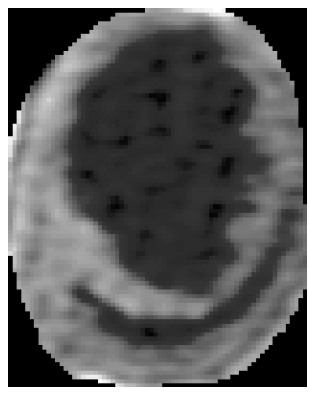

(c)

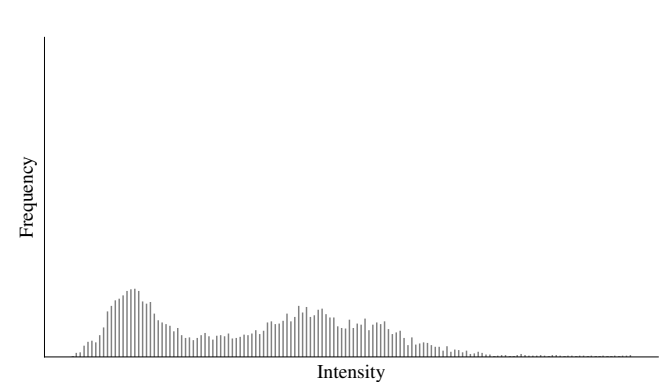

(b)

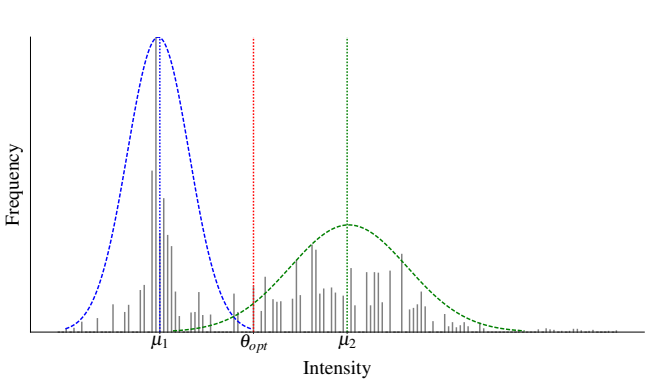

(d)

Figure 4: Enhanced image obtained by MedGA on an example of uterine fibroid (size: $89 \times 70$ pixels): (a) normalized input image using linear contrast stretching on the initial full range of the masked MR image; (c) resulting image after the application of the pre-processing using MedGA. The histograms corresponding to the sub-images in (a) and (c) are shown in (b) and (d), respectively. The final histogram emphasizes the two underlying distributions in the gray levels intensity characterized by mean values $\mu_{1}$ and $\mu_{2}$, and standard deviations $\sigma_{1}$ and $\sigma_{2}$, respectively. The two distributions are highlighted with blue and green dashed lines.

to take into account NPV inhomogeneities, due to sonication spots during the MRgFUS treatment 61].

The used post-processing refinement steps are the following (Fig. 3a):

1. morphological opening with a circular structuring element (2-pixel radius) to separate possible loosely connected hypo-intense regions;

2. some regions at the boundary of the uterus bounding region mask could present similar intensity values to gray levels characterizing fibroid regions, so being included in the thresholding output. To eliminate this ambigu- 


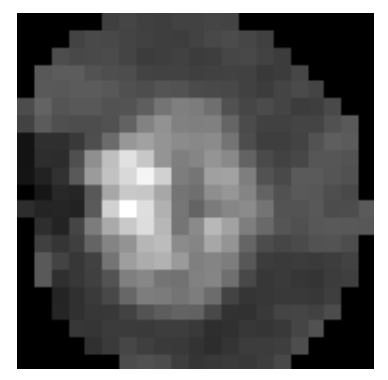

(a)

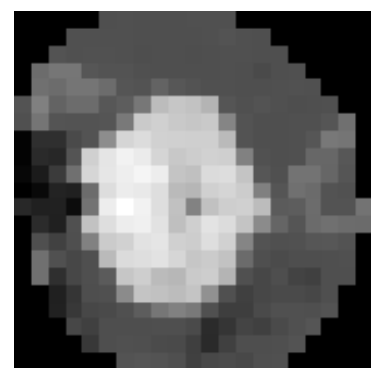

(c)

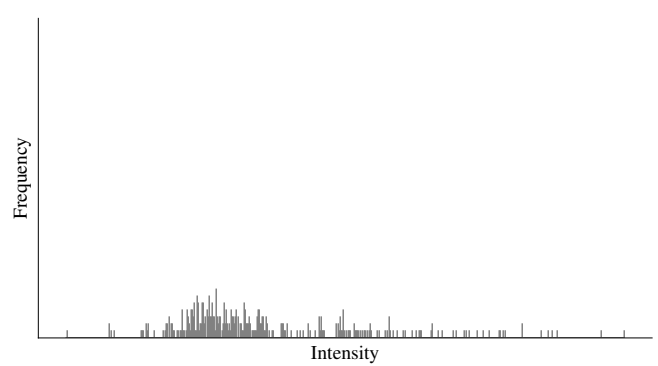

(b)

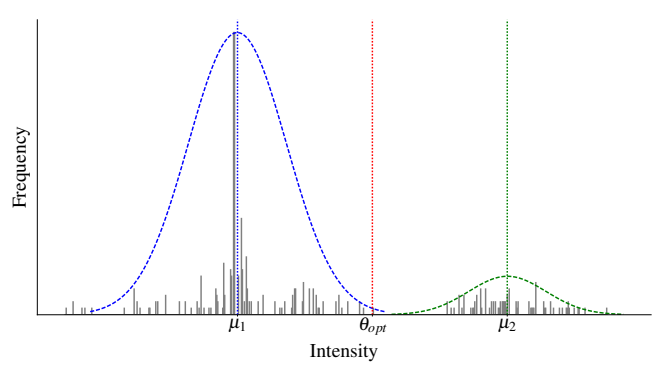

(d)

Figure 5: Enhanced image obtained by MedGA on an example of brain tumor (size: $21 \times 21$ pixels): (a) normalized input image using linear contrast stretching on the initial full range of the masked MR image; (c) resulting image after the application of the pre-processing using MedGA. The histograms corresponding to the sub-images in (a) and (c) are shown in (b) and (d), respectively. The final histogram emphasizes the two underlying distributions in the gray levels intensity characterized by mean values $\mu_{1}$ and $\mu_{2}$, and standard deviations $\sigma_{1}$ and $\sigma_{2}$, respectively. The two distributions are highlighted with blue and green dashed lines.

ity, it is appropriate to apply a morphological erosion (with a circular structuring element of 5-pixel radius) to the ROI binary mask, and then the logical pixel-by-pixel product (i.e., Hadamard multiplication) with the image resulting from the previous step is performed;

3. a hole filling algorithm is necessary to deal with possible holes in fibroid regions also due to non-uniform distribution of ablated tissue caused by sonication spots;

4. segmentation is further improved through a connected-component labeling based operation by removing objects that are smaller than a certain area 


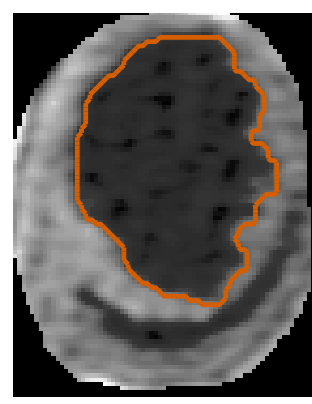

(a)

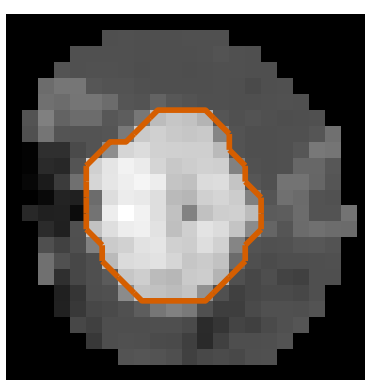

(b)

Figure 6: Segmentation results achieved by the IOTS algorithm on the MR images preprocessed by MedGA: (a) uterine fibroid delineation on the image given in Fig. 4c. (b) brain tumor delineation on the image given in Fig. $5 \mathrm{c}$

(i.e., 120 pixels) and characterized by similar intensity with respect to the fibroids to be treated, because there may be regions or artifacts caused by very small dark areas;

5. some lengthened connected-component with sufficiently large area could be present (i.e., due to other anatomical structures or to acquisition artifacts). Fibroids, in fact, present a spherical or semi-spherical shape 62] that can be denoted by means of the parameters of the various connectedcomponents. This connected-component based selection considers the eccentricity (that is, the ratio between the foci distance related to an ellipse and its own major axis length) and the extent (that is, the ratio between the pixels belonging to the region and the bounding box pixels) of the detected regions. Specifically, experimental reference values to discern fibroids from the rest of connected-components are: $0.3 \leq$ extent $<0.8$ and $0.0 \leq$ eccentricity $<0.8$, according to 60 . Lastly, any connectedcomponent, which has passed the shape-based control and whose centroid distance is more than a given upper limit (i.e., $\frac{\sqrt{M^{2}+N^{2}}}{3}$ ) from the MR image center, is removed.

Brain tumor segmentation. The accurate and reproducible measurement of tumor size and its changes over time is crucial for diagnosis, treatment planning, 
as well as monitoring of response to oncological therapy for brain tumors [23].

As a preliminary step, the user has to interactively select a bounding region that includes the tumor zone (by means of a free-hand "lasso" tool). Since the areas to segment are enhancement regions, the pixels that have higher intensities than the threshold are selected during the image binarization phase. Brain metastatic cancers may contain also necrotic cores, which could affect the achieved enhancement region segmentation. Therefore, some refinement steps are useful to cope with this situation.

The used post-processing pipeline is described in the following (see Fig. 3 3 ):

1. hole filling algorithm to consider also necrotic areas;

2. adaptive post-processing steps based on the size of the input image, consisting in small area removal (considering 4-connectivity) with minimum threshold equal to 30 pixels on images with size greater than 300 pixels, or 10 pixels otherwise;

3. to allow also for large bounding regions, shape-based selection is applied in the case of at least two connected-components, according to: extent $\geq 0.6$ and $0.0 \leq$ eccentricity $<0.8$ (see [56]). However, when a single connectedcomponent is present, these controls are avoided;

4. brain metastases have a pseudo-spherical appearance [63, therefore a convex hull algorithm is employed to envelope the segmented lesion into the smallest convex polygon containing this region.

\subsection{Existing image pre-processing methods}

We compared MedGA against the most common and popular image preprocessing techniques for image enhancement, namely:

- Histogram Equalization (HE) [7, which adjusts pixel intensities for contrast enhancement according to the normalized histogram of the original image $\mathcal{I}_{\text {orig. }}$ With HE, gray levels are more uniformly distributed on the histogram, by spreading the most frequent intensity values; 
- Bi-Histogram Equalization (Bi-HE) [33] - a modification of the traditional $\mathrm{HE}$ - which addresses issues concerning mean brightness preservation;

- Gamma Transformation (GT), which is a non-linear operation using the power-law relationship $s=c r^{\gamma}$, where $r$ and $s$ are the input and the output gray-scale values, respectively, and $c$ is a multiplication constant $(c=1$ in the following tests). The parameter $\gamma$ is set to values greater than 1 (i.e., decoding gamma) to obtain a gamma expansion, or to values smaller than 1 (i.e., encoding gamma) to realize a gamma compression. In our tests, we considered the values $\gamma=0.4$ and $\gamma=2.5$; higher (lower) values of $\gamma=2.5$ $(\gamma=0.4)$ tend to logarithmic (anti-logarithmic) functions, resulting in an excessively bright (dark) output image, unsuitable for practical medical applications [14;

- Sigmoid intensity Transformation (ST) function, also called S-shaped curve, which is a global non-linear mapping defined as follows:

$$
s(r)=\frac{l_{i n}^{(\max )}}{1+\exp (-\lambda(r-\alpha))},
$$

where $l_{\text {in }}^{(\max )}=\max \left\{\mathcal{L}_{i n}\right\}=\max \left\{\mathcal{L}_{\text {in }}^{\prime}\right\}$ is the asymptotic maximum value the function steepness. This transformation stretches the intensity around the level $\alpha$, by making the hypo-intense histogram part darker and the hyper-intense histogram part brighter. Thus, the difference between the minimum and maximum gray values as well as the gradient magnitude of the image are increased, obtaining strong edges [14. In our tests, we used sigmoid functions that allow for considering the entire input dynamic range, by varying the curve slope with the values $\lambda \in\left\{\frac{4}{\alpha}, \frac{6}{\alpha}, \frac{8}{\alpha}\right\}$.

\section{Experimental results}

This section presents the experimental results achieved by applying our com485 putational framework, considering the evaluation metrics for both image enhancement and segmentation briefly described in Supplementary Material. In 

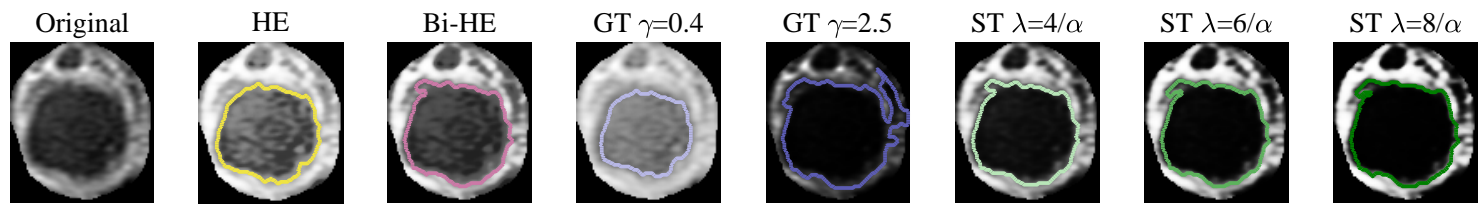

MedGA

(a)
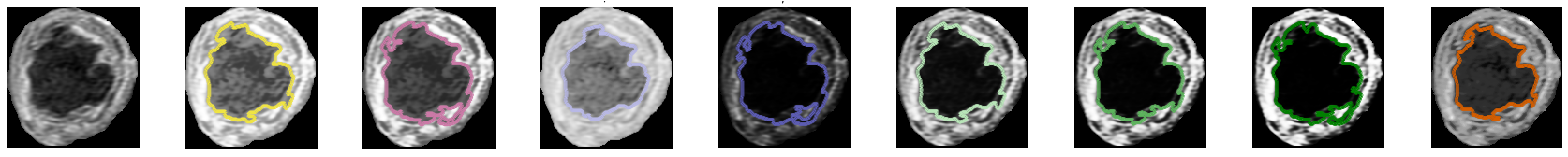

(b)

Figure 7: Segmentation results on the uterine fibroids shown in Figs. 2a and 2b achieved by the processing pipeline presented in Fig. 37 exploiting the state-of-the-art image preprocessing approaches (namely: HE, Bi-HE, GT $\gamma=2.5$, GT $\gamma=0.4$, ST $\lambda=4 / \alpha$, ST $\lambda=8 / \alpha, \mathrm{ST} \lambda=6 / \alpha)$ and MedGA.

particular, to achieve a comprehensive comparison between MedGA and the other pre-processing techniques listed above, we exploited the entire set of MRI data consisting in 18 patients affected by uterine fibroids and 27 brain metastatic cancers. Figs. 7 and 8 show two examples of uterine fibroid and brain tumor MR images, respectively, which were pre-processed by means of the comparison methods considered in this work and segmented by using the processing pipelines described in Sections 4.2.2.

\subsection{Medical image enhancement results}

Tables 2 and 3 show the image pre-processing results achieved by each method on the uterine fibroid and brain tumor MRI datasets, respectively. In both MR image analysis applications, MedGA remarkably obtains the highest Peak Signal-to-Noise Ratio $(P S N R)$ 64 mean values with respect to the state-of-the-art methods, generally involving the highest signal quality.

Considering the results in Table 2, HE over-enhances the processed uterine fibroid MR images, as denoted by the highest mean number of detected edges $(\# D E)$ value [65], while Bi-HE allows for the preservation of the mean brightness, as also indicated by the lowest mean value of Absolute Mean Brightness 

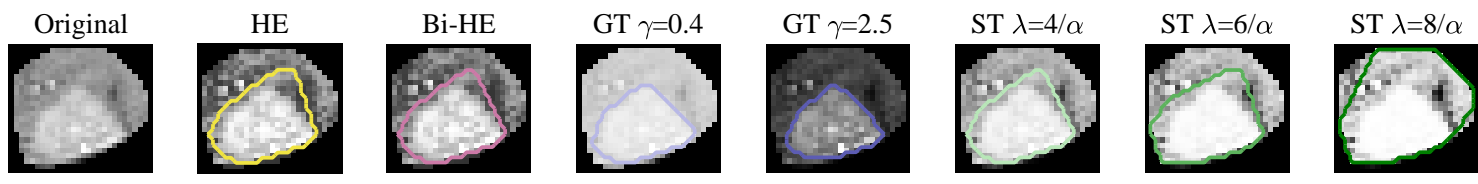

(a)
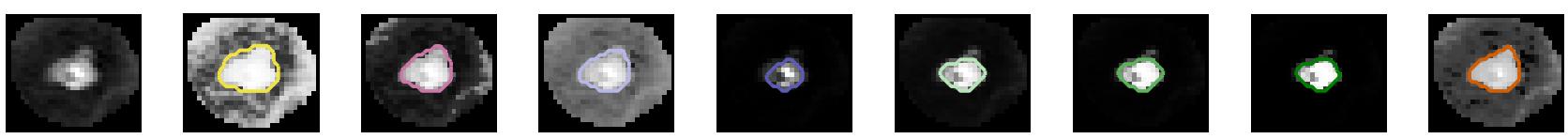

(b)

Figure 8: Segmentation results on the the brain tumors in Figs. 2c and 2d achieved by the processing pipeline in Fig. 3 3 by exploiting the state-of-the-art image pre-processing approaches (namely: HE, Bi-HE, GT $\gamma=2.5$, GT $\gamma=0.4$, ST $\lambda=4 / \alpha$, ST $\lambda=8 / \alpha$, ST $\lambda=6 / \alpha)$ and MedGA.

Error $(A M B E)$ [32, 66]. For what concerns the other techniques, on the one hand, GT with $\gamma=0.4$ yields better results compared to GT with $\gamma=2.5$, especially in the case of the Structural Similarity Index (SSIM) metrics [67; on the other hand, all metrics related to the tested ST functions show that their performances decrease as the value of $\lambda$ increases. As it can be observed in Figs. 7 and 8 , MedGA strengthens the ROI edges by enhancing details and features useful for image binarization; this result confirms, from a qualitative perspective, the quantitative results presented above. From an overall view of the metrics values, we can claim that the approaches obtaining the highest values of the \#DE measure (i.e., HE and GT with $\gamma=2.5$ ) could imply a considerable over-enhancement of the output image, according to the other image quality metrics.

The results on brain tumor MRI data reported in Table 3 show a slightly different trend, also due to the small size of the pre-processed cropped sub-images. As a first evidence, both GTs do not preserve the input mean brightness considering the $A M B E$ measure. Interestingly, GT with $\gamma=2.5$ and $\gamma=0.4$ achieve the highest and the lowest \#DE values, respectively. Bi-HE strongly improves the enhancement metrics obtained by HE, by generally reporting the 
Table 2: Values of the image enhancement evaluation metrics, achieved by applying the image pre-processing approaches considered in this work on the uterine fibroid MRI series, expressed as mean and standard deviation values over the 18 patients of the dataset. Numbers in bold indicate the best values.

\begin{tabular}{c|cc|cc|cc|cc}
\hline \hline \multirow{2}{*}{ Method } & \multicolumn{2}{|c|}{ PSNR } & \multicolumn{2}{c|}{ \#DE } & \multicolumn{2}{c|}{ AMBE } & \multicolumn{2}{c}{ SSIM } \\
& Mean & Std. Dev. & Mean & Std. Dev. & Mean & Std. Dev. & Mean & Std. Dev. \\
\hline HE & 30.994 & 1.949 & $\mathbf{9 7 5 . 4 6 5}$ & 475.951 & 0.085 & 0.029 & 0.859 & 0.044 \\
Bi-HE & 31.880 & 2.046 & 907.177 & 415.703 & 0.038 & 0.020 & 0.907 & 0.032 \\
GT $\gamma=0.4$ & 30.194 & 2.170 & 717.555 & 401.186 & 0.212 & 0.019 & 0.823 & 0.024 \\
GT $\gamma=2.5$ & 29.952 & 2.127 & 965.012 & 380.967 & 0.261 & 0.012 & 0.586 & 0.075 \\
ST $\lambda=4 / \alpha$ & 33.971 & 1.874 & 872.594 & 396.016 & 0.040 & 0.014 & 0.880 & 0.023 \\
ST $\lambda=6 / \alpha$ & 32.286 & 1.975 & 869.032 & 378.277 & 0.060 & 0.021 & 0.715 & 0.056 \\
ST $\lambda=8 / \alpha$ & 31.353 & 2.029 & 841.420 & 348.674 & 0.073 & 0.025 & 0.613 & 0.070 \\
MedGA & $\mathbf{3 7 . 3 6 6}$ & 2.347 & 866.604 & 409.604 & $\mathbf{0 . 0 3 3}$ & 0.011 & $\mathbf{0 . 9 2 8}$ & 0.025 \\
\hline \hline
\end{tabular}

Table 3: Values of the image enhancement evaluation metrics, achieved by applying the image pre-processing approaches considered in this work on the brain tumor MRI dataset, expressed as mean and standard deviation values over the 27 brain metastases. Numbers in bold indicate the best values.

\begin{tabular}{c|cc|cc|cc|cc}
\hline \hline \multirow{2}{*}{ Method } & \multicolumn{2}{|c|}{ PSNR } & \multicolumn{2}{c|}{ \#DE } & \multicolumn{2}{c|}{ AMBE } & \multicolumn{2}{c}{ SSIM } \\
& Mean & Std. Dev. & Mean & Std. Dev. & Mean & Std. Dev. & Mean & Std. Dev. \\
\hline HE & 34.215 & 1.447 & 38.779 & 21.287 & 0.124 & 0.049 & 0.756 & 0.112 \\
Bi-HE & 36.758 & 1.718 & 44.923 & 26.252 & $\mathbf{0 . 0 4 2}$ & 0.024 & $\mathbf{0 . 9 3 2}$ & 0.021 \\
GT $\gamma=0.4$ & 33.193 & 0.841 & 21.271 & 18.467 & 0.229 & 0.020 & 0.713 & 0.065 \\
GT $\gamma=2.5$ & 33.520 & 1.113 & $\mathbf{4 5 . 1 1 9}$ & 27.444 & 0.229 & 0.028 & 0.457 & 0.096 \\
ST $\lambda=4 / \alpha$ & 36.812 & 0.923 & 43.574 & 27.307 & 0.055 & 0.019 & 0.848 & 0.048 \\
ST $\lambda=6 / \alpha$ & 35.270 & 0.942 & 43.779 & 26.492 & 0.079 & 0.028 & 0.645 & 0.105 \\
ST $\lambda=8 / \alpha$ & 34.435 & 0.991 & 44.072 & 26.508 & 0.090 & 0.034 & 0.543 & 0.121 \\
MedGA & $\mathbf{3 7 . 7 5 1}$ & 1.990 & 43.534 & 22.598 & 0.079 & 0.039 & 0.881 & 0.053 \\
\hline \hline
\end{tabular}

best results. Consistently with the metrics calculated on uterine fibroid MRI data, the results concerning ST functions worsen as the value of $\lambda$ increases. The highest SSIM mean value is achieved by Bi-HE, revealing the best structural information, even though MedGA obtains the best signal quality in terms of PSNR mean values. These findings are also corroborated by a visual inspection of Figs. 7 and 8 , where the enhanced images using HE and GT with $\gamma=2.5$ present an inadequate appearance for image observation and interpretation. Overall, these results confirm that MedGA generally outperforms the conventional image enhancement approaches considering signal and perceived image quality, while preserving the input mean brightness. 

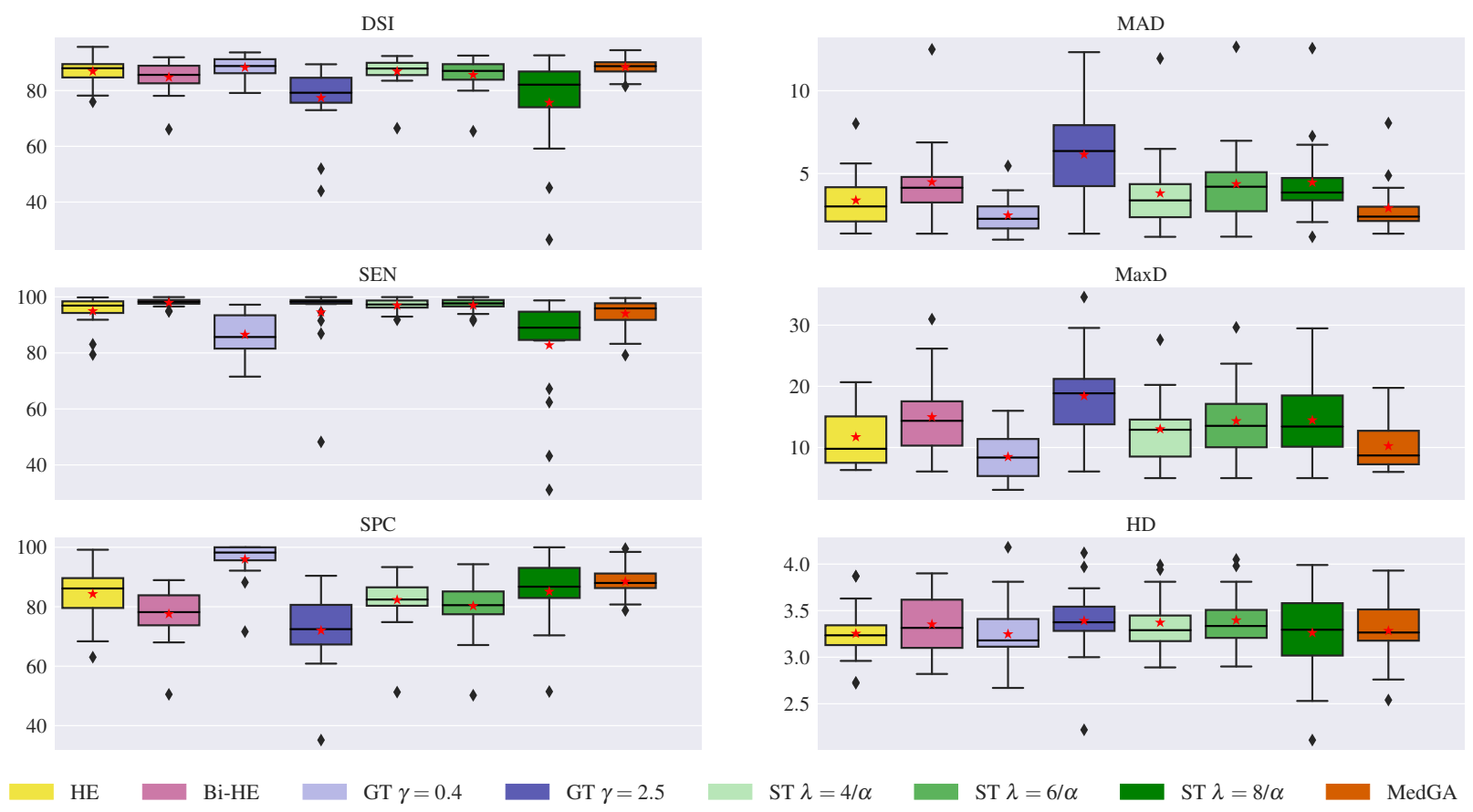

Figure 9: Boxplots of overlap-based and distance-based metrics (left and right columns, respectively) obtained on the MRI dataset composed of 18 patients affected by uterine fibroids who underwent MRgFUS treatment. The lower and the upper bounds of each boxplot represent the first and third quartiles of the distribution, respectively. The median and the mean values are represented by a black solid line and a red star, respectively. Whisker value is 1.5 in all cases, and outliers are displayed as black diamonds.

\subsection{Medical image segmentation results}

The quantitative segmentation results achieved by using the pipeline in Fig. 3a, employing the different pre-processing approaches, on the analyzed MRI dataset composed of 18 patients affected by uterine fibroids are depicted in the boxplots in Fig. 9, reporting both overlap-based and distance-based metrics values. Analogously, the boxplots concerning the segmentation results, achieved by using the pipeline in Fig. 3b on the analyzed MRI dataset consisting in 27 brain metastases, are shown in Fig. 10.

In the literature, it has been shown that a Dice Similarity Index $(D S I) 68$ 

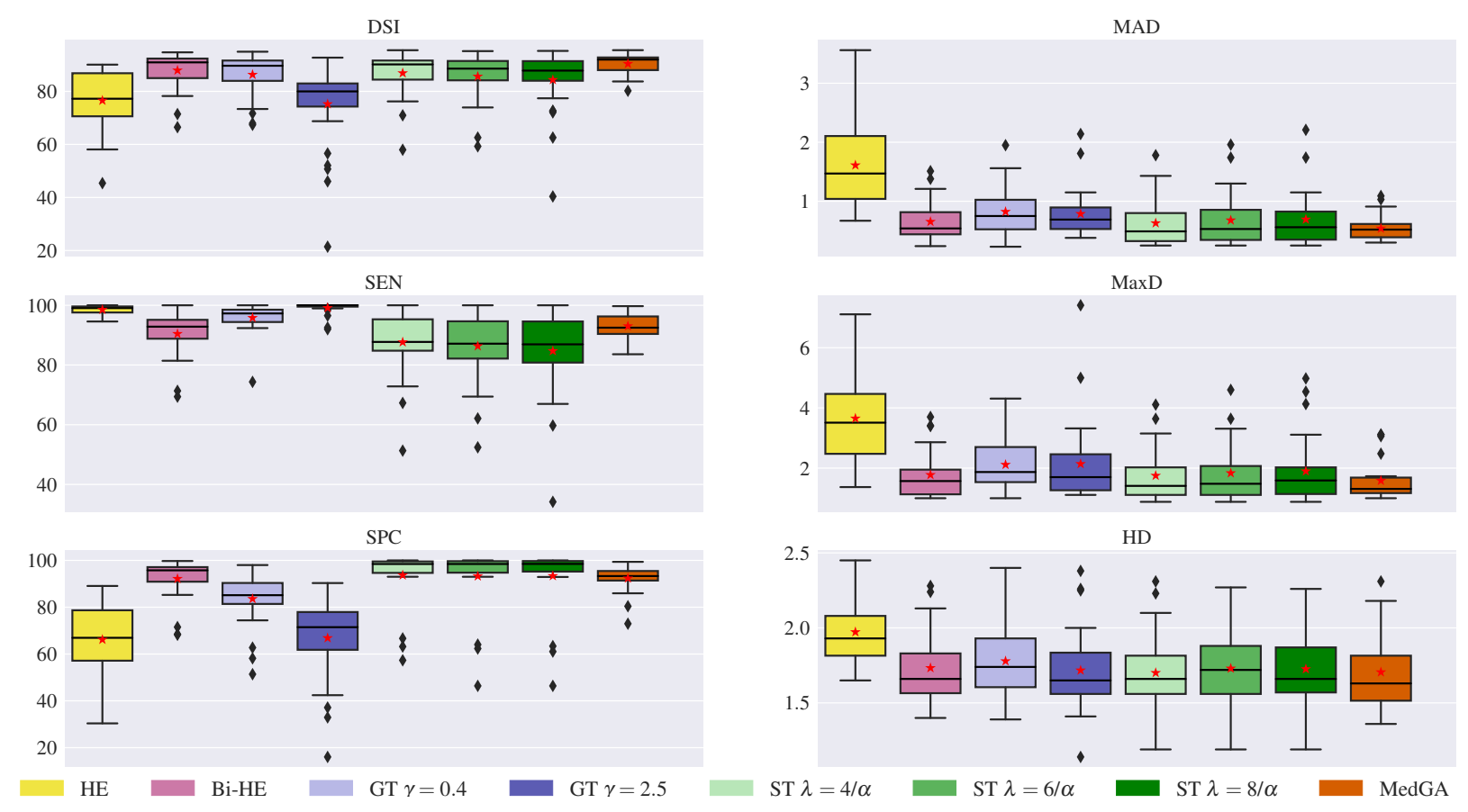

Figure 10: Boxplots of overlap-based and distance-based metrics (left and right columns, respectively) obtained on the MRI dataset composed of 27 brain metastatic tumors underwent stereotactic neuro-radiosurgery. The lower and the upper bounds of each boxplot represent the first and third quartiles of the distribution, respectively. The median and the mean values are represented by a black solid line and a red star, respectively. Whisker value is 1.5 in all cases, and outliers are displayed as black diamonds.

above $70 \%$ is generally regarded as a satisfactory level of agreement between two segmentations (i.e., manual and automated delineations) in clinical applications [69. Since the MR image segmentation methods proposed in Section 4.2 .2 obtain a $D S I$ appreciably higher than $70 \%$ regardless the pre-processing 545 technique, we can consider that the processing pipelines in Fig. 3 are clinically valuable, allowing for a fair comparison on segmentation performance among the state-of-the-art pre-processing algorithms.

In both cases, the segmentation results concerning the images pre-processed using MedGA achieved the highest mean and median DSI values, with low 
standard deviation. GT with $\gamma=0.4$ and Bi-HE obtained the second best performances for uterine fibroid and brain tumor MR image segmentation, respectively. We can thus claim that MedGA shows the highest accuracy and reliability in the two considered MRI analysis tasks. This is also confirmed by the boxplots, where the distributions for MedGA present significantly less than $10 \%$ outliers in all the overlap-based metrics, thus evidencing extremely low statistical dispersion. As a matter of fact, MedGA is the only technique that significantly supports the IOTS algorithm in both dark (i.e., uterine fibroid NPV) and bright (i.e., brain tumor enhancement region) ROI extraction. In agreement with the image enhancement results discussed $n$ Section 5.1 , GT with $\gamma=0.4$ considerably outperforms GT with $\gamma=2.5$. The decreasing trend, related to ST when the value of $\lambda$ increases, is also confirmed. ST with $\lambda=4 / \alpha$ achieved good results in both cases. Brain tumor MR images pre-processed by means of HE achieved low DSI values, but better results are obtained on uterine fibroid MR segmentation with respect to Bi-HE. Overall, the achieved segmentation performance shows the great accuracy and reliability of the proposed EC-based computational model. Considering sensitivity $(S E N)$ and specificity $(S P C)$ [70], MedGA yielded the best trade-off between these two often conflicting measures that should be always considered and combined together. These metrics reveal that the other techniques could involve over-segmentation or under-segmentation.

The achieved spatial distance-based indices (namely: Mean Absolute Distance $(M A D)$, Maximum absolute Distance $(M a x D)$, Hausdorff Distance $(H D)$ [70]) are consistent with overlap-based metrics, also observing the corresponding boxplots shown in Figs. 9 and 10 . Hence, MedGA allows also for accurate results in terms of distance between the automated and the manual boundaries. It is worth noting that, generally, the boxplots pertaining to MedGA results present the lowest statistical dispersion (in terms of box width and number of outliers), thus implying a lower standard deviation with respect to the conventional techniques. Therefore, the use of MedGA as a pre-processing step allows for considerably robust and reliable segmentation results. These experimental 


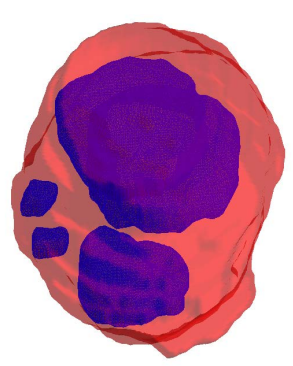

(a)

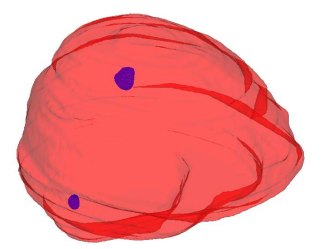

(c)

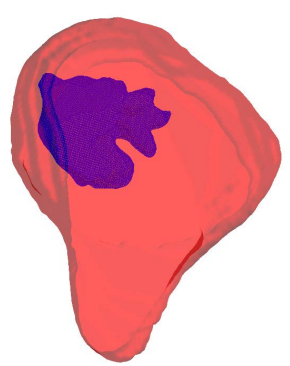

(b)

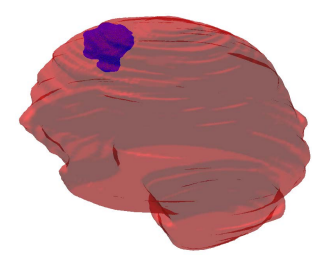

(d)

Figure 11: Tridimensional reconstruction of the segmented ROIs (blue volumetric models) in their real location with respect to the enclosing organ (transparent red surface): $(a, b)$ uterine fibroids within the uterus, segmented using the method in 60; (c, d) brain tumors within the whole brain, achieved using a skull stripping algorithm. Transparent surfaces are rendered with alpha blending $(\alpha=0.40)$

findings are endorsed by the segmentation examples shown in Figs. 7 and 8 .

Fig. 11 shows two pairs of examples of tridimensional reconstructions of the ROIs, i.e., uterine fibroids and brain tumors, permitting to display their actual locations in the whole uterus and brain (represented by means of a transparent red surface), respectively. This visualization allows for an intuitive and comprehensive representation of complex data [71].

\section{Discussion and conclusions}

In this paper, we proposed a novel intelligent framework designed ad hoc for enhancing MR image segmentation results of MRI data characterized by an underlying bimodal histogram. Our approach covers all the steps required for medical image analysis, including the quantification and radiology reporting phase 
(see Fig. 1). Our framework employs an evolutionary image pre-processing approach, called MedGA [18, which exploits a GA that aims at emphasizing bimodal histogram separation and, consequently, optimizing the subsequent seg-

The main limitation of the approach presented in this paper resides in the 
time required by the image enhancement pre-processing step, lasting approximately 2 minutes per image (running on a computational platform equipped with a 6-core Intel ${ }^{\circledR}$ Xeon ${ }^{\circledR}$ E5-2440 CPU at $2.40 \mathrm{GHz}, 16 \mathrm{~GB}$ RAM, and CentOS 7 operating system). As a matter of fact, MedGA has been currently used only for off-line image analysis. Our framework would certainly benefit from a porting of the current Python code into a faster compiled programming language (e.g., $\mathrm{C} / \mathrm{C}++$ ) 73

In the specific case of tomography image stack analysis, we developed a Master-Slave version of our framework to distribute on multiple CPU cores the computations pertaining to different slices 74, achieving a sub-linear speed-up with respect to the number of the available CPU cores. Consequently, by exploiting efficient programming languages and High-Performance Computing (HPC) paradigms, we can overcome the current limitations, making the pipelines presented in this work a clinically feasible solutions in real-time radiology applications.

In the near future, we plan to apply our framework also to other clinical contexts requiring MR image analysis and segmentation to provide useful insights for differential diagnosis and prognosis, such as in the case of breast cancer [75, 76] and meningiomas [77, also for differentiating tumor grade.

\section{Conflict of interest statement}

All authors in this paper have no potential conflict of interest.

\section{Acknowledgements}

We thank the Department of Biomedicine, Neuroscience and Advanced Diagnostics at the University of Palermo, Palermo, Italy, and the Cannizzaro Hospital, Catania, Italy, for their support and for supplying the images-uterine fibroids and metastatic brain tumors, respectively - analyzed in this study. 
This work was conducted in part using the resources of the Advanced Computing Center for Research and Education (ACCRE) at the Vanderbilt University, Nashville, TN, USA.

\section{References}

[1] I. N. Bankman, Part II - Segmentation, in: I. N. Bankman (Ed.), Handbook of Medical Image Processing and Analysis, 2nd Edition, Academic Press, Burlington, MA, USA, 2009, pp. 71-72. doi:10.1016/ B978-012373904-9.50008-8.

[2] S. Wang, R. M. Summers, Machine learning and radiology, Med. Image Anal. 16 (5) (2012) 933-951. doi:10.1016/j.media.2012.02.005.

[3] A. João, A. M. Gambaruto, J. Tiago, A. Sequeira, Computational advances applied to medical image processing: an update, Open Access Bioinform. 2016 (8) (2016) 1-15. doi:10.2147/OAB.S70327

[4] J. S. Duncan, N. Ayache, Medical image analysis: progress over two decades and the challenges ahead, IEEE Trans. Pattern Anal. Mach. Intell. 22 (1) (2000) 85-106. doi:10.1109/34.824822.

[5] T. E. Yankeelov, D. A. Mankoff, L. H. Schwartz, F. S. Lieberman, J. M. Buatti, J. M. Mountz, B. J. Erickson, F. M. M. Fennessy, W. Huang, J. Kalpathy-Cramer, et al., Quantitative imaging in cancer clinical trials, Clin. Cancer Res. 22 (2) (2016) 284-290.

[6] R. J. Gillies, P. E. Kinahan, H. Hricak, Radiomics: images are more than 670 pictures, they are data, Radiology 278 (2) (2015) 563-577. doi:10.1148/ radiol.2015151169.

[7] R. C. Gonzalez, R. E. Woods, Digital Image Processing, 3rd Edition, Prentice Hall Press, Upper Saddle River, NJ, USA, 2002. 
[8] J. Rogowska, Chapter 5 - Overview and fundamentals of medical image segmentation, in: I. N. Bankman (Ed.), Handbook of Medical Image Processing and Analysis, 2nd Edition, Academic Press, Burlington, MA, USA, 2009, pp. 73-90. doi:10.1016/B978-012373904-9.50008-8.

[9] J. H. Xue, Y. J. Zhang, Ridler and Calvard's, Kittler and Illingworth's and Otsu's methods for image thresholding, Pattern Recognit. Lett. 33 (6) (2012) 793-797. doi:10.1016/j.patrec.2011.01.021.

[10] R. H. Daffner, Visual illusions in computed tomography: phenomena related to Mach effect, Am. J. Roentgenol. 134 (2) (1980) 261-264. doi: 10.2214/ajr.134.2.261.

[11] E. A. Krupinski, Current perspectives in medical image perception, Atten. Percept. Psychophys. 72 (5) (2010) 1205-1217. doi:10.3758/APP.72.5. 1205.

[12] F. Ratliff, Why Mach bands are not seen at the edges of a step, Vision Res. 24 (2) (1984) 163-165. doi:10.1016/0042-6989(84)90102-0.

[13] C. Li, Y. Yang, L. Xiao, Y. Li, Y. Zhou, J. Zhao, A novel image enhancement method using fuzzy Sure entropy, Neurocomputing 215 (2016) 196-211. doi:10.1016/j.neucom.2015.07.156.

[14] A. Gandhamal, S. Talbar, S. Gajre, A. F. M. Hani, D. Kumar, Local gray level S-curve transformation-a generalized contrast enhancement technique

1 for medical images, Comput. Biol. Med. 83 (2017) 120-133. doi:10.1016/ j.compbiomed.2017.03.001

[15] P. M. Evans, Anatomical imaging for radiotherapy, Phys. Med. Biol. 53 (12) (2008) R151. doi:10.1088/0031-9155/53/12/R01.

[16] J. G. Sled, A. P. Zijdenbos, A. C. Evans, A nonparametric method for automatic correction of intensity nonuniformity in MRI data, IEEE Trans. Med. Imaging 17 (1) (1998) 87-97. doi:10.1109/42.668698. 
[17] T. B. Smith, MRI artifacts and correction strategies, Imaging Med. 2 (4) (2010) 445-457. doi:10.2217/iim.10.33

[18] L. Rundo, A. Tangherloni, M. S. Nobile, C. Militello, D. Besozzi, G. Mauri, P. Cazzaniga, MedGA: a novel evolutionary method for image enhancement 705 ㅁ in medical imaging systems, Expert Syst. Appl. 119 (2019) 387-399. doi: $10.1016 /$ j.eswa.2018.11.013.

[19] T. W. Ridler, S. Calvard, Picture thresholding using an iterative selection (1) method, IEEE Trans. Syst. Man Cybern. 8 (8) (1978) 630-632. doi:10. 1109/TSMC.1978.4310039.

[20] H. J. Trussell, Comments on picture thresholding using an iterative selection method, IEEE Trans. Syst. Man Cybern. 9 (5) (1979) 311-311. doi:10.1109/TSMC.1979.4310204.

[21] N. Dey, A. S. Ashour, S. Beagum, D. S. Pistola, M. Gospodinov, E. P. Gospodinova, J. M. R. S. Tavares, Parameter optimization for local polynomial approximation based intersection confidence interval filter using genetic algorithm: an application for brain MRI image de-noising, J. Imaging 1 (1) (2015) 60-84. doi:10.3390/jimaging1010060.

[22] C. Militello, L. Rundo, M. C. Gilardi, Applications of imaging processing to MRgFUS treatment for fibroids: a review, Transl. Cancer Res. 3 (5) (2014) 472-482. doi:10.3978/j.issn.2218-676X.2014.09.06.

[23] R. Meier, U. Knecht, T. Loosli, S. Bauer, J. Slotboom, R. Wiest, M. Reyes, Clinical evaluation of a fully-automatic segmentation method for longitudinal brain tumor volumetry, Sci. Rep. 6. doi:10.1038/srep23376

[24] M. Sonka, V. Hlavac, R. Boyle, Image Processing, Analysis, and Machine Vision, Thomson-Engineering, 2007.

[25] K. Suzuki, I. Horiba, N. Sugie, Neural edge enhancer for supervised edge enhancement from noisy images, IEEE Trans. Pattern Anal. Mach. Intell. 25 (12) (2003) 1582-1596. doi:10.1109/TPAMI.2003.1251151. 
[26] M. Šprogar, M. Šprogar, M. Colnarič, Autonomous evolutionary algorithm in medical data analysis, Comput. Methods Programs Biomed. 80 (2005) S29-S38. doi:10.1016/S0169-2607(05) 80004-5.

[27] J. L. Garcia-Arroyo, B. Garcia-Zapirain, Segmentation of skin lesions in dermoscopy images using fuzzy classification of pixels and histogram thresholding, Comput. Methods Programs Biomed. 168 (2019) 11-19. doi:10.1016/j.cmpb.2018.11.001

[28] L. Shamir, J. D. Delaney, N. Orlov, D. M. Eckley, I. G. Goldberg, Pattern recognition software and techniques for biological image analysis,

a PLoS Comput. Biol. 6 (11) (2010) e1000974. doi:10.1371/journal.pcbi. 1000974

[29] K. D. Toennies, Guide to Medical Image Analysis, 3rd Edition, SpringerVerlag, London, UK, 2017. doi:10.1007/978-1-4471-7320-5

[30] L. Xiong, H. Li, L. Xu, An enhancement method for color retinal images based on image formation model, Comput. Methods Programs Biomed. 143 (2017) 137-150. doi:10.1016/j.cmpb.2017.02.026.

[31] P. K. Sahoo, S. Soltani, C. Y. C. Wong, A. K. C., A survey of thresholding techniques, Comput. Vis. Graph. Image Process. 41 (2) (1988) 233-260. doi:10.1016/0734-189X(88)90022-9.

[32] S. D. Chen, A. R. Ramli, Minimum mean brightness error bi-histogram equalization in contrast enhancement, IEEE Trans. Consumer Electron. 49 (4) (2003) 1310-1319. doi:10.1109/TCE.2003.1261234.

[33] Y. T. Kim, Contrast enhancement using brightness preserving bi-histogram equalization, IEEE Trans. Consumer Electron. 43 (1) (1997) 1-8. doi: $10.1109 / 30.580378$

[34] B. Xiao, H. Tang, Y. Jiang, W. Li, G. Wang, Brightness and contrast controllable image enhancement based on histogram specification, Neurocomputing 275 (2018) 2798-2809. doi:10.1016/j.neucom.2017.11.057. 
[35] B. Shi, X. Bai, C. Yao, An end-to-end trainable neural network for imagebased sequence recognition and its application to scene text recognition, IEEE Trans. Pattern Anal. Mach. Intell. 39 (11) (2017) 2298-2304. doi: 10.1109/TPAMI . 2016.2646371.

[36] W. Zhou, L. Zhang, L. Jiao, Linear programming support vector machines, Pattern Recognit. 35 (12) (2002) 2927-2936. doi:10.1016/ S0031-3203(01)00210-2.

[37] A. Ortiz, . J. Górriz, J. Ramírez, D. Salas-Gonzalez, J. M. Llamas-Elvira, Two fully-unsupervised methods for MR brain image segmentation using SOM-based strategies, Appl. Soft Comput. 13 (5) (2013) 2668-2682. doi: $10.1016 /$ j.asoc. 2012.11 .020 .

[38] Z. Ma, J. M. R. S. Tavares, R. M. N. Jorge, A review on the current segmentation algorithms for medical images, in: Proc. 1st International Conference on Imaging Theory and Applications (IMAGAPP), 2009.

[39] P. C. T. Gonçalves, J. M. R. S. Tavares, R. M. N. Jorge, Segmentation and simulation of objects represented in images using physical principles,

n Comput. Model. Eng. Sci. 32 (1) (2008) 45-55. doi:10.3970/cmes. 2008. 032.045

[40] M. J. M. Vasconcelos, J. M. R. S. Tavares, Methods to automatically build point distribution models for objects like hand palms and faces represented

प in images, Comput. Model. Eng. Sci. 36 (3) (2008) 213-241. doi:10.3970/ cmes.2008.036.213.

[41] A. Ferreira, F. Gentil, J. M. R. S. Tavares, Segmentation algorithms for ear image data towards biomechanical studies, Comput. Methods Biomech.

1. Biomed. Eng. 17 (8) (2014) 888-904. doi:10.1080/10255842.2012. 723700 .

[42] Z. Ma, J. M. R. S. Tavares, R. N. Jorge, T. Mascarenhas, A review of algorithms for medical image segmentation and their applications to the female 
pelvic cavity, Comput. Methods Biomech. Biomed. Eng. 13 (2) (2010) 235246. doi:10.1080/10255840903131878.

[43] R. B. Oliveira, E. Mercedes Filho, Z. Ma, J. P. Papa, A. S. Pereira, J. M. R. S. Tavares, Computational methods for the image segmentation of pigmented skin lesions: a review, Comput. Methods Programs Biomed. 131 (2016) 127-141. doi:10.1016/j.cmpb.2016.03.032Get

[44] J. H. Holland, Adaptation in Natural and Artificial Systems: An Introductory Analysis with Applications to Biology, Control and Artificial Intelligence, MIT Press, Cambridge, MA, USA, 1992.

[45] N. Otsu, A threshold selection method from gray-level histograms, IEEE Trans. Syst. Man Cybern. 11 (285-296) (1975) 23-27. doi:10.1109/TSMC. 1979.4310076

[46] J. Kittler, J. Illingworth, Minimum error thresholding, Pattern Recognit. 19 (1) (1986) 41-47. doi:10.1016/0031-3203(86)90030-0.

[47] Q. Z. Ye, P. E. Danielsson, On minimum error thresholding and its 800 implementations, Pattern Recognit. Lett. 7 (4) (1988) 201-206. doi: 10.1016/0167-8655(88)90103-1.

[48] T. Kurita, N. Otsu, N. Abdelmalek, Maximum likelihood thresholding based on population mixture models, Pattern Recognit. 25 (10) (1992) 1231-1240. doi:10.1016/0031-3203(92)90024-D.

[49] X. Xu, S. Xu, L. Jin, E. Song, Characteristic analysis of Otsu threshold 【 and its applications, Pattern Recognit. Lett. 32 (7) (2011) 956-961. doi: $10.1016 / j \cdot$ patrec.2011.01.021.

[50] R. Medina-Carnicer, R. Muñoz-Salinas, A. Carmona-Poyato, F. J. MadridCuevas, A novel histogram transformation to improve the performance of thresholding methods in edge detection, Pattern Recognit. Lett. 32 (5) (2011) 676-693. doi:10.1016/j.patrec.2010.12.012. 
[51] S. M. Kwok, R. Chandrasekhar, Y. Attikiouzel, M. T. Rickard, Automatic pectoral muscle segmentation on mediolateral oblique view mammograms, IEEE Trans. Med. Imaging 23 (9) (2004) 1129-1140. doi:10.1109/TMI. 2004.830529

[52] A. Roberts, Magnetic resonance-guided focused ultrasound for uterine fibroids, Semin. Interv. Radiol. 25 (4) (2008) 394. doi:10.1055/ s-0028-1102999.

[53] L. Rundo, C. Militello, S. Vitabile, C. Casarino, G. Russo, M. Midiri, M. C. Gilardi, Combining split-and-merge and multi-seed region growing algorithms for uterine fibroid segmentation in MRgFUS treatments, Med. Biol. Eng. Comput. 54 (7) (2016) 1071-1084. doi:10.1007/ s11517-015-1404-6.

[54] L. Leksell, The stereotaxic method and radiosurgery of the brain, Acta Chir. Scand. 102 (4) (1951) 316-319.

[55] L. Rundo, C. Militello, G. Russo, S. Vitabile, M. C. Gilardi, G. Mauri, GTVcut for neuro-radiosurgery treatment planning: an MRI brain cancer seeded image segmentation method based on a cellular automata model, Nat. Comput. 17 (3) (2018) 521-536. doi:10.1007/s11047-017-9636-z.

[56] L. Rundo, A. Stefano, C. Militello, G. Russo, M. G. Sabini, C. D’Arrigo, F. Marletta, M. Ippolito, G. Mauri, S. Vitabile, M. C. Gilardi, A fully automatic approach for multimodal PET and MR image segmentation in Gamma Knife treatment planning, Comput. Methods Programs Biomed. 144 (2017) 77-96. doi:10.1016/j.cmpb.2017.03.011

[57] L. Rundo, C. Militello, A. Tangherloni, G. Russo, S. Vitabile, M. C. Gilardi, G. Mauri, NeXt for neuro-radiosurgery: a fully automatic approach for necrosis extraction in brain tumor mri using an unsupervised machine learning technique, Int. J. Imaging Syst. Technol. 28 (1) (2018) 21-37. doi:10.1002/ima.22253. 
860

[64] S. Hashemi, S. Kiani, N. Noroozi, M. E. Moghaddam, An image contrast enhancement method based on genetic algorithm, Pattern Recognit. Lett. 31 (13) (2010) 1816-1824. doi:10.1016/j.patrec.2009.12.006.

[65] J. Canny, A computational approach to edge detection, IEEE Trans. Pat865 tern Anal. Mach. Intell. PAMI-8 (6) (1986) 679-698. doi:10.1109/TPAMI. 1986.4767851 
[66] E. F. Arriaga-Garcia, R. E. Sanchez-Yanez, M. G. Garcia-Hernandez, Image enhancement using bi-histogram equalization with adaptive sigmoid functions, in: Proc. IEEE International Conference on Electronics, Communications and Computers (CONIELECOMP), IEEE, 2014, pp. 28-34. doi:10.1109/CONIELECOMP.2014.6808563

[67] Z. Wang, A. C. Bovik, H. R. Sheikh, E. P. Simoncelli, Image quality assessment: from error visibility to structural similarity, IEEE Trans. Image Proc. 13 (4) (2004) 600-612. doi:10.1109/TIP.2003.819861.

${ }_{875}^{6}$ [68] K. H. Zou, S. K. Warfield, A. Bharatha, C. M. C. Tempany, M. R. Kaus, S. J. Haker, W. M. Wells, F. A. Jolesz, R. Kikinis, Statistical validation of image segmentation quality based on a spatial overlap index, Acad. Radiol. 11 (2) (2004) 178-189. doi:10.1016/S1076-6332(03)00671-8.

[69] A. P. Zijdenbos, B. M. Dawant, R. A. Margolin, A. C. Palmer, Morphometric analysis of white matter lesions in MR images: method and validation, IEEE Trans. Med. Imaging 13 (4) (1994) 716-724. doi: $10.1109 / 42.363096$

[70] A. A. Taha, A. Hanbury, Metrics for evaluating 3D medical image segmentation: analysis, selection, and tool, BMC Med. Imaging 15 (1) (2015) 29. doi : 10.1186/s12880-015-0068-x.

[71] T. Walter, D. W. Shattuck, R. Baldock, M. E. Bastin, A. E. Carpenter, S. Duce, J. Ellenberg, A. Fraser, N. Hamilton, S. Pieper, et al., Visualization of image data from cells to organisms, Nat. Methods 7 (2010) S26-S41. doi:10.1038/nmeth.1431.

[72] A. Draa, A. Bouaziz, An artificial bee colony algorithm for image contrast enhancement, Swarm Evol. Comput. 16 (2014) 69-84. doi:10.1016/j. Swevo.2014.01.003

[73] S. Behnel, R. Bradshaw, C. Citro, L. Dalcin, D. S. Seljebotn, K. Smith, 
Cython: the best of both worlds, Comput. Sci. Eng. 13 (2) (2011) 31-39. doi:10.1109/MCSE.2010.118.

[74] J. Pinho, J. L. Sobral, M. Rocha, Parallel evolutionary computation in bioinformatics applications, Comput. Methods Programs Biomed. 110 (2) (2013) 183-191. doi:10.1016/j.cmpb.2012.10.001.

[75] J. Anitha, J. D. Dinesh Peter, S. Immanuel Alex Pandian, A dual stage adaptive thresholding (DuSAT) for automatic mass detection in mammograms, Comput. Methods Programs Biomed. 138 (2017) 93-104. doi: $10.1016 / \mathrm{j} . \mathrm{cmpb} \cdot 2016 \cdot 10.026$

[76] M. Banaie, H. Soltanian-Zadeh, H.-R. Saligheh-Rad, M. Gity, Spatiotemporal features of DCE-MRI for breast cancer diagnosis, Comput. Methods 905 \ Programs Biomed. 155 (2018) 153-164. doi:10.1016/j.cmpb.2017.12. 015.

[77] A. T. Hale, L. Wang, M. K. Strother, L. B. Chambless, Differentiating meningioma grade by imaging features on magnetic resonance imaging, J. Clin. Neurosci. 48 (2018) 71-75. doi:10.1016/j.jocn.2017.11.013. 